\title{
Desempenho operacional de um pulverizador costal elétrico ${ }^{1}$
}

\author{
Robson Shigueaki Sasaki², Mauri Martins Teixeira², \\ Luís Eduardo Nogueira ${ }^{2}$, Cleyton Batista de Alvarenga ${ }^{3}$, Marcus Vinicius Morais de Oliveira ${ }^{4}$
}

\begin{abstract}
Operational performance of

an electrical backpack sprayer

The knapsack sprayer is a very popular equipment, due to its low acquisition cost and wide applicability. In recent years, electric backpack sprayers, which increase the operator's comfort and improve the spraying operation quality, have come up. This study aimed at evaluating an electric backpack sprayer, by using four flat spraying nozzles (XR 110.02, AXI 110.03, AXI 110.04 and AXI 110.05). The variables studied were: liquid flow and pressure, battery charge duration, axis rotation and pump noise. The electric sprayer provided constant flow and pressure for the XR 110.02, AXI 110.03 and AXI 110.04 nozzles. By using the AXI 110.05 nozzle, a variation was observed for the liquid flow and pressure, mainly at the end of each repetition, since the flow increase also increased the pump power demand. Similarly, the battery charge duration was proportional to the power demand. For the flows studied, the pump noise fitted the regulation standards, for a daily working journey of 8 hours.
\end{abstract}

KEY-WORDS: Hydraulic spraying; flat spraying nozzles; liquid flow and pressure.

O pulverizador costal manual é um equipamento muito empregado por pequenos, médios e grandes produtores, dependendo da necessidade na propriedade, devido ao seu baixo custo e ampla aplicabilidade de uso, tanto com distintos produtos, como em diversas culturas e infestações localizadas ou em área total (Freitas 2006). Um dos grandes problemas na utilização deste equipamento é o esforço físico repetitivo e o despreparo da maioria dos aplicadores para calibrá-lo.

Nos últimos anos, tem-se observado o surgimento de vários equipamentos costais elétricos no

\section{RESUMO}

O pulverizador costal manual é um equipamento bastante utilizado, devido ao seu baixo custo de aquisição e ampla aplicabilidade. Nos últimos anos, têm surgido pulverizadores costais elétricos que aumentam o conforto do operador e melhoram a qualidade da operação de pulverização. Objetivouse, com este trabalho, avaliar um pulverizador costal elétrico, utilizando-se quatro pontas de pulverização de jato plano (XR 110.02, AXI 110.03, AXI 110.04 e AXI 110.05). As variáveis estudadas foram: vazão e pressão do líquido, duração da carga da bateria, rotação do eixo e ruído da bomba. O pulverizador elétrico proporcionou vazão e pressão constantes para as pontas XR 110.02, AXI 110.03 e AXI 110.04. Já utilizando-se a ponta AXI 110.05, houve variação na vazão e pressão do líquido, principalmente no final de cada repetição, uma vez que, com o aumento da vazão, aumentou a demanda de potência da bomba. Da mesma forma, a duração da carga da bateria foi proporcional à demanda de potência. Nas vazões estudadas, o ruído da bomba atendeu aos padrões da legislação, para uma jornada de 8 horas de trabalho.

PALAVRAS-CHAVE: Pulverização hidráulica; pontas de jato plano; vazão e pressão de líquido.

mercado, visando a aumentar o conforto do trabalhador, pois não necessitam do acionamento manual da alavanca e, aparentemente, apresentam baixos níveis de ruído próximo ao ouvido do operador.

Independentemente do sistema de pulverização, é importante determinar a intensidade do ruído. Aybek et al. (2010) afirmam que o ruído é considerado um dos principais fatores que afetam a saúde e a eficiência dos trabalhadores. Os efeitos nocivos dos ruídos não se restringem somente à audição, mas podem ocasionar distúrbios emocionais, cardiovasculares, fadiga e estresse (Silveira et al. 2007).

1. Trabalho recebido em mar./2013 e aceito para publicação em set./2013 (n ${ }^{\circ}$ registro: PAT 20236).

2. Universidade Federal de Viçosa (UFV), Departamento de Engenharia Agrícola, Viçosa, MG, Brasil. E-mails: robsonsasaki@yahoo.com.br,mauri@ufv.br, luis.nogueira@ufv.br.

3. Universidade Federal de Mato Grosso (UFMT), Instituto de Ciências Agrárias, Sinop, MT, Brasil. E-mail: cleytonbatista@yahoo.com.br.

4. Universidade Federal Rural do Rio de Janeiro (UFRRJ), Departamento de Engenharia Agrícola, Seropédica, RJ, Brasil. E-mail: marvin_moroli@yahoo.com.br. 
Os pulverizadores costais elétricos têm incorporado novas tecnologias que garantem maior controle da pressão de trabalho e do volume de aplicação, por meio da automatização dos equipamentos. Em uma aplicação de produto fitossanitário, a pressão de trabalho deverá ser constante, pois está diretamente relacionada à qualidade da pulverização.

Cunha \& Silva (2010) constataram efeito da pressão de trabalho no perfil de distribuição de líquido. Cunha (2007) e Sasaki et al. (2011) observaram que a uniformidade de distribuição de líquido foi afetada pela altura e pressão de trabalho. Fernandes et al. (2007) verificaram que o tamanho das gotas foi influenciado pela pressão de trabalho. Ferreira et al. (2007) detectaram que a pressão de trabalho influenciou na vazão e no ângulo formado pelo jato de pulverização.

Diante do exposto, objetivou-se, com este trabalho, avaliar um pulverizador costal elétrico, quanto aos parâmetros pulverização, vazão e pressão do líquido, e, também, quanto à duração da carga da bateria, rotação do eixo e ruído da bomba, utilizando-se quatro pontas de pulverização de jato plano (XR 110.02, AXI 110.03, AXI 110.04 e AXI 110.05).

$\mathrm{O}$ trabalho foi conduzido no Laboratório de Mecanização Agrícola da Universidade Federal de Viçosa, Campus de Viçosa (MG), em 2011, onde as condições de temperatura variaram entre $23^{\circ} \mathrm{C} \mathrm{e}$ $28^{\circ} \mathrm{C}$ e a umidade relativa do ar entre $65 \%$ e $75 \%$, com ausência de ventos.

Utilizou-se, para o estudo, um pulverizador elétrico da marca MTS, modelo Spritz $18^{\circledR}$, que é constituído por um depósito com capacidade para $18 \mathrm{~L}$, uma bomba de diafragma acionado por um motor elétrico (potência de $30 \mathrm{~W}$ ) e sensor de pressão do tipo diferencial (precisão de 2,5\% e tempo de resposta de $1 \mathrm{~s}$ ). A fonte de energia para o equipamento é constituída por uma bateria chumbo-ácida de $12 \mathrm{~V}$, com capacidade de carga de $9 \mathrm{Ah}$.

Todos os ensaios realizados com o pulverizador costal elétrico utilizaram quatro modelos de pontas de pulverização hidráulica de jato plano (XR 110.02, AXI 110.03, AXI 110.04 e AXI 110.05).

Preliminarmente, as pontas utilizadas nos ensaios foram aferidas, quanto à vazão, perfil e uniformidade de distribuição de líquido. Nestes ensaios, as pontas foram montadas em uma barra porta-bicos, sobre uma mesa de ensaios padronizada, de acordo com a norma ISO 5682/1 (ISO 1986). Esta bancada é dotada de um manômetro, que foi previamente ca- librado. Todos os ensaios foram realizados à pressão de $300 \mathrm{kPa}$. Nos testes de uniformidade de distribuição de líquido, os ensaios foram conduzidos a uma altura do alvo e espaçamento entre pontas de $0,5 \mathrm{~m}$. Determinou-se, ainda, o coeficiente de variação (CV).

Posteriormente, a fim de verificar a duração da bateria e os parâmetros da pulverização, padronizou-se o tempo de carregamento em 15 horas. Com a bateria carregada, cada ponta foi acoplada ao pulverizador costal elétrico, o qual permaneceu ligado até o completo descarregamento. A cada cinco minutos, com o auxílio de um manômetro, aferido e acoplado à lança do pulverizador, verificou-se a pressão do líquido, e, com uma proveta graduada com precisão de $10 \mathrm{~mL}$, determinou-se a vazão. Coletou-se, também, a cada cinco minutos, a tensão da bateria, com o auxílio de um multímetro (marca Minipa, modelo ET 2030A), e a corrente elétrica, utilizando-se um segundo multímetro (marca Minipa, modelo ET 2510).

Para caracterizar a rotação da bomba, utilizou-se um tacômetro digital (marca Minipa, modelo MDT $2238 \mathrm{~A}^{\circledR}$ ), e, para o ruído, um decibelímetro digital (marca Minipa, modelo MSL $1350^{\circledR}$ ). O nível de ruído foi avaliado a uma distância de $0,3 \mathrm{~m}$ do equipamento, nas proximidades do ouvido de um operador, e os resultados obtidos foram confrontados com a norma NR-15 (Brasil 2011).

Todo o experimento foi realizado em delineamento inteiramente casualizado, com quatro tratamentos (pontas hidráulicas). Na caracterização das pontas de pulverização, os dados foram representados por meio de estatística descritiva, com três repetições por tratamento. Já no ensaio com o pulverizador, as variáveis dependentes foram a duração da bateria, vazão e pressão de líquido, comportamento da bateria (tensão e corrente), rotação da bomba e nível de ruído, com quatro repetições por tratamento.

Quando pertinente, as médias dos dados foram comparadas pelo teste Tukey, ou por meio de análise de regressão, com nível de significância de $5 \%$, em ambos os casos. Antes de proceder à análise de variância, foram verificadas a homogeneidade de variância e normalidade dos erros, pelos testes de Cochran e Lilliefors, respectivamente.

Nos ensaios com as pontas, verificou-se que as vazões nominais, para os modelos XR 110.02, AXI 110.03, AXI 110.04 e AXI 110.05, foram de $0,78 \mathrm{~L} \mathrm{~min}^{-1}, 1,17 \mathrm{~L} \mathrm{~min}^{-1}, 1,52 \mathrm{~L} \mathrm{~min}^{-1}$ e $1,97 \mathrm{~L} \mathrm{~min}^{-1}$, respectivamente, estando todas em conformidade com a norma ISO 10625 (ISO 2005). Quanto ao perfil 
de distribuição, todas as pontas apresentaram perfil típico de uma ponta leque, sendo que a amplitude total da faixa de pulverização variou de $1,35 \mathrm{~m}$ a $1,45 \mathrm{~m}$, para as pontas AXI 110.03 e AXI 110.05, respectivamente. $\mathrm{Na}$ avaliação da uniformidade de distribuição do líquido, a ponta XR 110.02 apresentou coeficiente de variação de $13,24 \%$. Para as pontas AXI 110.03, AXI 110.04 e AXI 110.05, os coeficientes de variação obtidos foram de $9,62 \%, 9,51 \%$ e $8,54 \%$, respectivamente.

O conjunto de dados técnicos proporcionados pelas pontas é importante, visto que determina a qualidade da ponta e, consequentemente, da pulverização. A vazão nominal, bem como a uniformidade de distribuição, é um indicativo da qualidade e do estado de conservação das pontas. De certa forma, padrões rigorosos, com valores baixos de coeficiente de variação, representam distribuição uniforme de líquido sobre a área tratada.

$\mathrm{Na}$ avaliação do pulverizador costal elétrico, quanto à pressão e vazão de líquido, ao longo do tempo, observou-se que as pontas XR 110.02, AXI 110.03 e AXI 110.04 apresentaram variação praticamente nula, na pressão e vazão, indicando que, independentemente do nível de carga da bateria, o pulverizador proporcionou pressões e vazões constantes. Quando se utilizou a ponta AXI 110.05, ocorreu pequena variação na pressão e vazão da ponta: no início dos ensaios a pressão foi de $300 \mathrm{kPa}$, reduzindo-se para $251 \mathrm{kPa}$, ao final das repetições (Figura 1).

Esses resultados demonstram que o equipamento possui grande aplicabilidade em campo, uma vez que proporciona pressão constante, e, logo, mantém a qualidade em uma pulverização.

Quanto à duração da bateria, houve aumento na demanda de potência da bomba, à medida em que aumentou a vazão, reduzindo-se, assim, o tempo de trabalho do equipamento. Quando utilizou-se a ponta XR 110.02, a duração da bateria foi de $340 \mathrm{~min}$., diferindo, estatisticamente, pelo teste Tukey, a 5\%, dos demais tratamentos, que apresentaram duração de 193,3 min., 166,7 min. e 161,7 min., para as pontas AXI 110.03, AXI 110.04 e AXI 110.05, respectivamente.

A utilização de baixo volume de calda é recomendada para aumentar a capacidade operacional dos equipamentos agrícolas. Uma alternativa para se reduzir o volume de pulverização é o uso de pontas de menor vazão.
Observou-se que a duração da carga da bateria está diretamente relacionada à queda de tensão, ao longo do tempo (Figura 2). Constatou-se que, com o aumento da vazão, houve maior gasto de energia.

Avaliando-se o comportamento da corrente, para as pontas XR 110.02, AXI 110.03, AXI 110.04 e AXI 110.05, a corrente média foi de 1,13 A, 1,62 A, 1,93 A e 2,32 A, respectivamente.

O aumento da vazão proporcionou maior demanda de potência da bomba, com aumento na rotação e nível de ruído (Figura 3). Os valores de ruído permaneceram abaixo do estabelecido pela NR-15. Para a maior vazão $\left(1,97 \mathrm{~L} \mathrm{~min}^{-1}\right)$, o ruído foi de 77 dB. O limite de tolerância para ruído contínuo ou

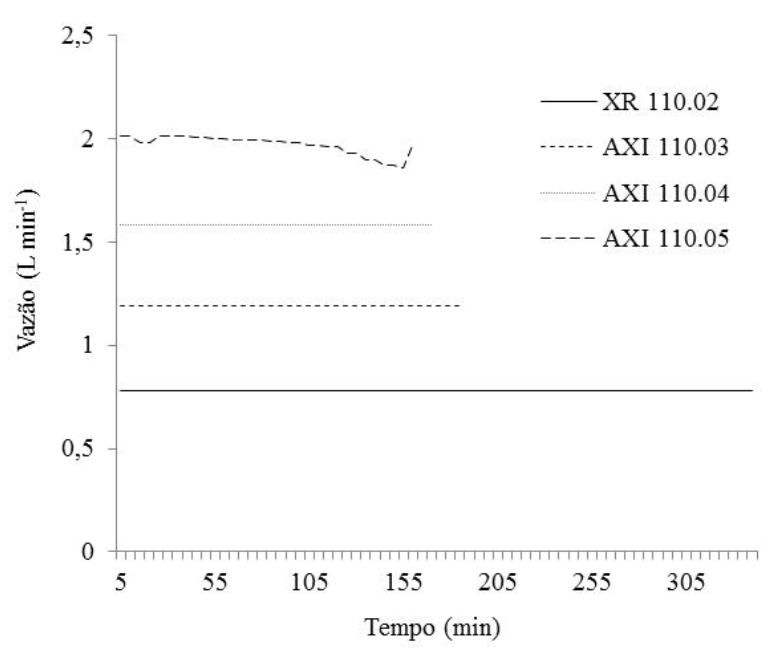

Figura 1. Comportamento da vazão das pontas, ao longo do tempo (Viçosa, MG, 2011).

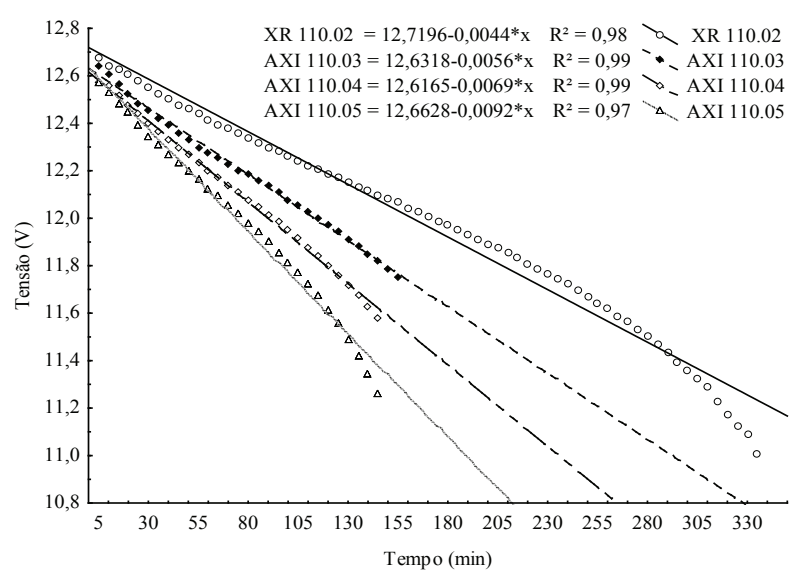

Figura 2. Relação entre tensão da bateria e tempo de funcionamento do equipamento (Viçosa, MG, 2011). * Significativo a $5 \%$, pelo teste $\mathrm{t}$. 


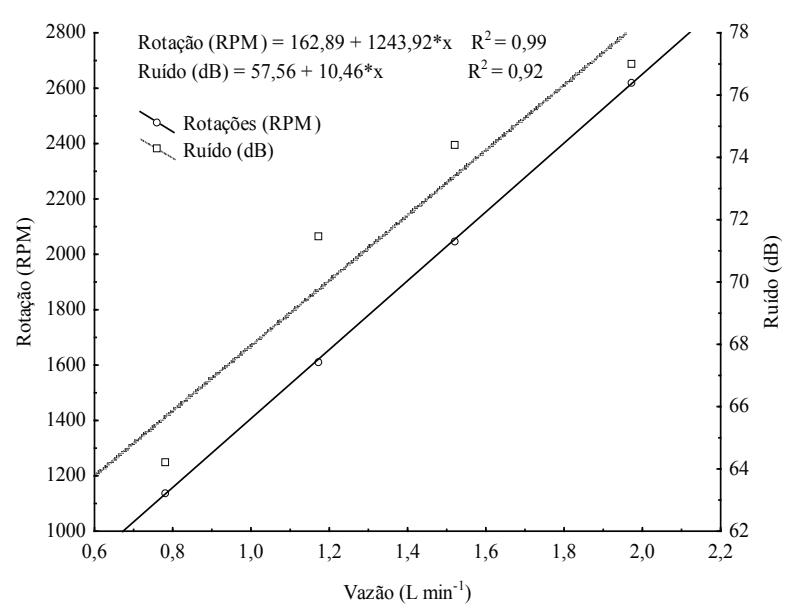

Figura 3. Rotação da bomba e nível de ruído junto ao ouvido do operador, conforme alteração na vazão (Viçosa, MG, 2011). * Significativo a 5\%, pelo teste t.

intermitente é de $85 \mathrm{~dB}$, para uma jornada de 8 horas (Brasil 2011). Cunha \& Teodoro (2006), avaliando pulverizadores motorizados portáteis, observaram níveis de ruído acima de $100 \mathrm{~dB}$, ou seja, acima do limite tolerado pela norma regulamentadora.

Diante dos resultados, concluiu-se que o pulverizador costal elétrico avaliado apresentou pressão constante, ao longo dos testes, para as pontas XR 110.02, AXI 110.03 e AXI 110.04. A duração da bateria é inversamente proporcional ao aumento da taxa de aplicação. O pulverizador não apresentou problemas de ruído, estando em conformidade com a norma NR-15.

\section{REFERÊNCIAS}

AYBEK, A. et al. Personal noise exposures of operators of agricultural tractors. Applied Ergonomics, Madison, v. 41, n. 2, p. 274-281, 2010.

BRASIL. Ministério do Trabalho e Emprego. Atividades e operações insalubres (NR-15). 2011. Disponível em: <http://www.mte.gov.br/legislacao/normas regulamentadoras/nr_31.pdf $>$. Acesso em: 20 jan. 2011.

CUNHA, J. P. A. R. Análise da distribuição volumétrica de pontas de pulverização hidráulicas de jato plano. Revista de Ciências Agrárias, Belém, v. 31, n. 1, p. 233-239, 2007.

CUNHA, J. P. A. R.; SILVA, R. A. M. Uniformidade de distribuição volumétrica de pontas de pulverização em função da pressão de trabalho e altura da barra. Bioscience Journal, Uberlândia, v. 26, n. 1, p. 52-58, 2010.
CUNHA, J. P. A. P.; TEODORO, R. E. F. Avaliação do nível de ruído em derriçadores e pulverizadores motorizados portáteis utilizados em lavouras de café. Bioscience Journal, Uberlândia, v. 22, n. 3, p. 71-77, 2006.

FERNANDES, A. P. et al. Caracterização do perfil de deposição e do diâmetro de gotas e otimização do espaçamento. Engenharia Agrícola, Jaboticabal, v. 27, n. 3, p. 728-733, 2007.

FERREIRA, M. C. et al. Fatores qualitativos da ponta de energia hidráulica ADGA 110015. Engenharia Agrícola, Jaboticabal, v. 27, n. 2, p. 471-478, 2007.

FREITAS, C. S. Análise ergonômica da atividade com pulverizador costal manual na cultura do café no municipio de Caratinga - MG. 2006. 70 f. Dissertação (Mestrado em Meio Ambiente e Sustentabilidade) - Centro Universitário de Caratinga, Caratinga, 2006.

INTERNATIONAL ORGANIZATION FOR STANDARDIZATION (ISO). Equipment for crop protection - spraying equipment. Part 2: Test methods for agricultural sprayers (ISO 5682/2). Geneva: ISO, 1986.

INTERNATIONAL ORGANIZATION FOR STANDARDIZATION (ISO). Equipment for crop protection - sprayer nozzles - colour coding for identification (ISO 10625). Geneva: ISO, 2005.

SASAKI, R. S. et al. Uniformidade de distribuição volumétrica de pontas de pulverização hidráulica em diferentes condições operacionais. Engenharia na Agricultura, Viçosa, v. 19, n. 6, p. 541-547, 2011.

SILVEIRA, J. C. M. et al. Níveis de ruído em função do raio de afastamento emitido por diferentes equipamentos em uma oficina agrícola. Engenharia na Agricultura, Viçosa, v. 15, n. 1, p. 66-74, 2007. 